REMARK. If $X_{1}, X_{2}$ are two continuous curves and $\rho_{i}: X_{i} \rightarrow I, i=1,2$, are monotone mappings onto, then $S\left(X_{1}, X_{2}, \rho_{1}, \rho_{2}\right)$ need not be locally connected.

\title{
REFERENCES
}

1. C. E. Capel, Inverse limit spaces, Duke Math. J. vol. 21 (1954) pp. 233-246.

2. H. Hahn, Mengentheoretische Charakterisierung der stetigen Kurve, Sitzungsber. Akad. Wiss., Wien, vol. 123 (1914) pp. 2433-2489.

3. J. L. Kelley, General topology, New York, van Nostrand Co., 1955. 209.

4. S. Mazurkiewicz, Sur les lignes de Jordan, Fund. Math. vol. 1 (1920) pp. 166-

5. R. L. Wilder, Topology of manifolds, Amer. Math. Soc. Colloquium Series, vol. 32, New York, 1949.

The University of Zagreb, Zagreb, Yugoslavia

\section{A CLAN WITH ZERO WITHOUT THE FIXED POINT PROPERTY}

\section{HASKELL COHEN}

There is a conjecture due to A. D. Wallace that a clan (i.e., a compact, connected, topological semigroup with identity element) with a zero element has the fixed point property. This is related to another conjecture of Wallace that a compact connected topological lattice has the fixed point property [4]. A proof of the latter conjecture for the finite dimensional case has recently been given by Dyer and Shields [1]. There is an example due to Kinoshita [2] of a contractable continuum without the fixed point property. The purpose of this note is to exhibit a multiplication which will make Kinoshita's example into a clan with zero, and, thus, provide a counter example to the first conjecture above.

We exhibit first a result which seems to be rather generally known, but which, to the author's knowledge, does not appear in print.

Lemma. Suppose $S$ is a topological semigroup, and $f$ is an open or closed map taking $S$ onto $T$, a Hausdorff space. Suppose further that $f(a)=f(b)$ and $f(c)=f(d)$ implies $f(a c)=f(b d)$. Then $T$ can be given $a$ multiplication which makes it a topological semigroup and which makes f a homomorphism.

Presented to the Society, November 19, 1960; received by the editors January 29, 1960. 
Proof. For $t_{1}$ and $t_{2}$ in $T$ we define $t_{1} \cdot t_{2}$ as follows. Let $a_{1}$ and $a_{2}$ be elements of $S$ such that $f\left(a_{1}\right)=t_{1}$ and $f\left(a_{2}\right)=t_{2}$. Let $t_{1} \cdot t_{2}=f\left(a_{1} a_{2}\right)$. It is easily seen that the multiplication is well defined and associative, and that $f$ is a homomorphism. The only item which remains to be checked is the continuity of the multiplication. Let $m: T \times T \rightarrow T$ be defined by $m\left(t_{1}, t_{2}\right)=t_{1} \cdot t_{2}$. We need only show that $m$ is continuous. If $f$ is an open map, let $P$ be an open set in $T$ (if $f$ were closed, we would, of course, take $P$ closed). Then $m^{-1}(P)=\left\{\left(t_{1}, t_{2}\right) \mid t_{1} \cdot t_{2} \in P\right\}$ $=\left\{\left(t_{1}, t_{2}\right) \mid f\left[f^{-1}\left(t_{1}\right) f^{-1}\left(t_{2}\right)\right] \in P\right\}$ is seen to be open since $f$ and multiplication in $S$ are continuous and $f$ is open. Thus $m$ is continuous completing the proof.

Now let

$$
\begin{aligned}
& A=\{(r, \theta, z) \mid 0 \leqq r<1, z=0\} \\
& B=\left\{(r, \theta, z) \mid r=\frac{2}{\pi} \tan ^{-1} \theta, \theta \geqq 0,0 \leqq z \leqq 1\right\},
\end{aligned}
$$

and

$$
C=\{(r, \theta, z) \mid r=1,0 \leqq z \leqq 1\},
$$

where $r, \theta$, and $z$ represent the usual cylindrical coordinates in three space. Let $K=A \cup B \cup C$, then $K$ is the continuum of the example of Kinoshita. For a continuous function on $K$ to $K$ which moves every point, the reader is referred to Kinoshita [2].

Let $D$ be the projection of $B \cup C$ into the $z=0$ plane. Define a multiplication "o" on $D$ by

$$
\left(r_{1}, \theta_{1}\right) \circ\left(r_{2}, \theta_{2}\right)=\left[\max \left\{r_{1}, r_{2}, \frac{2}{\pi} \tan ^{-1}\left(\theta_{1}+\theta_{2}\right)\right\}, \theta_{1}+\theta_{2}\right] .
$$

It is easy to see that " $\circ$ " is associative and continuous and that $(0,0)$ is an identity element. It, perhaps, should be mentioned that $D$, in slightly different form, is a rather well known clan (see e.g. the example on page 286 of [3]).

Now let $I$ be the interval $[0,1]$ with the usual multiplication, and let $E=D \times I$ with the coordinatewise multiplication; i.e., $\left(r_{1}, \theta_{1}, z_{1}\right)$ $\cdot\left(r_{2}, \theta_{2}, z_{2}\right)=\left[\left(r_{1}, 0_{1}\right) \circ\left(r_{2}, 0_{2}\right), z_{1} z_{2}\right]$.

Let $f: E \rightarrow R^{3}$ be defined by

$$
f(r, \theta, z)=(r, \theta, z) \text { if } r \leqq 2 z
$$

and

$$
f(r, \theta, z)=(2 z, \theta, z) \text { if } r \geqq 2 z .
$$


Let $F=f(E)$. It is easily seen that $f$ is continuous and, since $E$ is compact, that $f$ is closed. Let $p_{i}$ be the points $\left(r_{i}, \theta_{i}, z_{i}\right), i=1,2,3,4$, and suppose $f\left(p_{1}\right)=f\left(p_{2}\right)$ and $f\left(p_{3}\right)=f\left(p_{4}\right)$. We want to show $f\left(p_{1} p_{3}\right)$ $=f\left(p_{2} p_{4}\right)$. If $p_{1}=p_{2}$ and $p_{3}=p_{4}$, the result is clear. Hence suppose, say, $p_{1} \neq p_{2}$. Then $f\left(p_{1}\right)=f\left(p_{2}\right)$ implies $r_{1} \geqq 2 z_{1}, r_{2} \geqq 2 z_{2}, \theta_{1}=\theta_{2}$, and $z_{1}=z_{2}$. Now the $r$ coordinate of $p_{1} p_{3} \geqq r_{1} \geqq 2 z_{1} \geqq 2 z_{1} z_{3}$ and similarly the $r$ coordinate of $p_{2} p_{4} \geqq 2 z_{2} z_{4}$. Hence $f\left(p_{1} p_{3}\right)=\left(2 z_{1} z_{3}, \theta_{1}+\theta_{3}, z_{1} z_{3}\right)$ $=\left(2 z_{2} z_{4}, \theta_{2}+\theta_{4}, z_{2} z_{4}\right)=f\left(p_{2} p_{4}\right)$. Thus there is induced on $F$ the multiplication described in the lemma. With respect to this multiplication the points $(0,0,1)$ and $(0,0,0)$ are respectively the identity element and zero of $F$. Moreover it is clear that $F$ is homeomorphic to $K$. For example, the function $h$ defined by $h(r, \theta, z)=(r, \theta,(1-r / 2) z+r / 2)$ takes $K$ homeomorphically onto $F$. Applying the lemma again, $h^{-1}$ induces a multiplication which makes $K$ a clan with zero as was to be shown.

\section{REFERENCES}

1. Eldon Dyer and Allen Shields, Connectivity of topological lattices, Pacific J. Math. vol. 9 (1959) pp. 443-448.

2. Shin'ichi Kinoshita, On some contractible continua without fixed point property, Fund. Math. vol. 40 (1953) pp. 96-98.

3. R. J. Koch and A. D. Wallace, Admissibility of semigroup structures on continua, Trans. Amer. Math. Soc. vol. 88 (1958) pp. 277-287.

4. A. D. Wallace, Research Problem No. 9, Bull. Amer. Math. Soc. vol. 61 (1955) p. 93.

Louisiana State University 